\title{
Optimal Hedging: Managing Time and Cost Uncertainty in the Stochastic Time-Cost-Quality Tradeoff Project Scheduling Problem
}

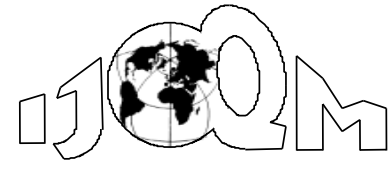

DOI: $10.46970 / 2021.27 .1 .1$

Volume 27, Number 1

March 2021, pp.1-21
Bruce Pollack-Johnson

Matthew J. Liberatore

Villanova University

(bruce.pollack-johnson@villanova.edu)

(matthew.liberatore@villanova.edu)

We consider how uncertainty affects tradeoffs between time, cost, and quality, modeling quality as a continuous function of time and cost. A translation agency example demonstrates that uncertainty can be modeled by making the quality function parameters stochastic. This leads to our Stochastic Time Cost Quality Tradeoff Problem (STCQTP), which determines optimal levels of time, cost, and quality to maximize expected quality. A software development example applies this model to show the considerable impact uncertainty can have on project quality. In addition, multiple probability distributions are considered, and the effect of correlation between time and cost is explored. The STCQTP model provides an approach to determine an optimal hedge against uncertainties that fully takes quality into consideration.

Keywords: Project Management, Quality, Project Scheduling, Uncertainty, Hedging, Stochastic Optimization

\section{Introduction}

Seasoned project managers know that delays and cost overruns are more common than completion on time and within budget. Successful project managers are able to hedge for these uncertainties intuitively, keeping quality considerations in mind. Conceptually, how could we define an optimal hedge against uncertainties of time and cost? This paper will present an approach to address this question.

Project management is "the application of knowledge, skills, tools, and techniques to project activities to meet the project requirements." (Project Management Institute, 2017). It consists of five phases: initiating, planning, executing, monitoring and controlling, and closing. The three key objectives of project management relate to completion time, total cost, and overall quality (Demeulemeester et al., 2006). In project management, "Quality management has equal priority with cost and schedule management" (Kerzner, 2003). This statement makes intrinsic sense, since project management is concerned with not only managing schedule and cost, but also the actual work needed to achieve the project goal. The quality of the work then is an important project outcome, since it directly relates to the value of the project deliverables (Liberatore and Pollack-Johnson, 2013).

From the earliest days when the discipline of project management was being developed, methods were created to assist in project planning, including the 
development of the critical path method (CPM) for project scheduling (Kelly and Walker, 1959). CPM allows managers to understand the tradeoff between project time and cost, but assumes quality is held constant. However, allocating additional time and money to a task can in many instances increase its quality. For example, in completing a building foundation there are choices related to the excavation depth and the compressive strength of the concrete used. These choices can lead to different levels of quality associated with a task.

More recently, researchers have realized that since the quality of project tasks is an important objective in managing a project, quality should be explicitly considered and included in project planning and scheduling models. This realization has led to a stream of research that addresses how to evaluate the tradeoffs between quality, cost, and time in project decision making. This approach requires that quality be measured for each project task.

Uncertainty related to project completion time and cost long have been considered using PERT (Malcolm et al., 1959) and simulation, as well as through newer approaches to assess the risks associated with project delays (Pfeifer et al., 2015; Floyd et al., 2017). No previous research has addressed the impact of uncertainty relating to task quality and its effect on a project plan and schedule. In this paper we extend Liberatore and Pollack-Johnson (2013) by considering how uncertainty, modeled by assigning a probability distribution to the task quality function parameters, affects the level of project quality and the project plan. We propose a stochastic optimization model to determine the optimal levels of time, cost, and quality when the quality function parameters are uncertain. Our analysis shows that this uncertainty can have a significant impact on the level of project quality achieved and the plan developed. Our proposed method provides a procedure for determining an optimal hedging strategy against these uncertainties.

In the next section, we review the literature on the time-cost-quality tradeoff problem (TCQTP). In section 3 we present a deterministic time-cost-quality tradeoff model as the baseline plan. In section 4 we show how uncertainty related to task time can affect the optimal level of project quality achieved using a translator project example, leading to our general formulation of the Stochastic Time Cost Quality Tradeoff Problem (STCQTP). In section 5 we apply the STCQTP model to show the effect of time and cost uncertainty on a software development project. Several probability distributions are considered, and the effect of correlation between time and cost is explored. Through this example, we illustrate how the STCQTP model provides an optimal hedging strategy against uncertainties of time and cost, with significant improvement in quality. In section 6 we draw some conclusions and discuss areas for future research.

\section{Literature Review}

As discussed in their review of the research on TCQTP, Orm and Jeunet (2018) characterized the literature as falling into three groups: rework as an implicit assessment of task quality (rework), quality as an estimated parameter in each execution mode for a task (discrete), and quality as a continuous function of its task duration only or its task duration and cost (continuous). The rework literature stream assumes that if a task does not fit all the requirements then rework is needed (IcmeliTukel and Rom, 1997; Kim et al., 2012; Tiwari et al., 2009). The problem objective 
is to minimize rework cost or rework time or a combination of both. The possibility of a range of quality levels for a task are not considered. In an earlier review Ghodsi et al. (2009) only considered the discrete and continuous models, presumably because the rework literature addresses quality in a limited manner.

In the discrete methods stream, different modes of completing a given activity are defined, and for each mode distinct values of quality, time, and cost are determined. The objective is to select a specific mode for each project task. Several methods have been proposed for this problem and have been reviewed in Tavana et al. (2014) and Orm and Jeunet (2018). As discussed in Orm and Jeunet (2018) the approaches for measuring task quality vary, with some authors estimating: quality for each mode (Tareghian and Taherei, 2006, 2007; Afruzi et al., 2014), quality associated with duration reduction (Mohammadipour and Sadjadi, 2016), and as a weighted sum of measurable quality indicators (El-Rayes and Kandil, 2005; Mungle et al., 2013; Monghasemi et al., 2015; Abshar et al., 2007),

In the continuous methods stream, quality is expressed as a continuous decreasing function of time only or as a nonlinear function of time and cost (Orm and Jeunet, 2018). Babu and Suresh (1996) and Khang and Myint (1999) make a direct subjective assessment of task quality. They use a linear scale for quality anchored with the normal and crash quality values for each activity, as a function of time only (similar to cost being a function of time only). Project quality in Babu and Suresh (1996) is measured as the arithmetic or geometric mean of the task quality values or as the minimum of the task qualities. The results of three mathematical programming models are used to evaluate the tradeoffs for quality, time and cost. Khang and Myint (1999) apply Babu and Suresh (1996) to a cement factory example. Similar linear scales for quality anchored at the normal and crash times are used by other authors, including Ghodsi et al. (2009) and Ahadian et al. (2016).

Several authors have applied modeling techniques such as fuzzy logic (Amiri and Golozari, 2011; Cristobol, 2013); grey numbers (Razavi et al., 2015; Mahdiraji et al., 2016); and genetic algorithms (Hosseini et al., 2018) to address this problem when quality follows a linear scale. Salmasnia et al. (2016) use a response surface methodology to find an optimal amount of resources to assign to activities so that project total time and cost are minimized while quality characteristics are maximized.

Zang et al. (2014) model task quality and cost as quadratic functions of task duration. Their approach assumes that after a threshold is reached, longer processing times degrade task quality. Project quality is measured as the arithmetic mean of the individual task qualities. The problem objective is to minimize a weighted sum of cost, time and quality differences as compared to contractual values. Zang et al. (2014) apply particle swarm optimization to solve this problem. Tran et al. (2015) follow Zang et al. (2014) in modeling task quality as a quadratic function of time but model cost as a linear decreasing function of time. These authors formulate their problem as a multi-objective program that seeks to minimize project makespan and cost and to maximize project quality. They employ an artificial bee colony with differential evolution as their solution approach.

Liberatore and Pollack-Johnson (2013) introduce the notion of a quality function and use the functional form of the bivariate normal distribution to model quality at the task level. The quality function is incorporated into a nonlinear programming 
model to evaluate the tradeoffs between quality, time, and cost. Their examples define overall project quality as the minimum of the individual task qualities (the weakest link concept), but the approach could easily be adapted for arithmetic or geometric mean or weighted average objectives.

$\mathrm{Fu}$ and Zhang (2016) follow Liberatore and Pollack-Johnson (2013) and use the bivariate normal function to model task quality. These authors include an additional parameter in the quality function to adjust the surface shape. They also partition the tasks into several groups to determine cumulative quality to allow a task's quality to depend not only on its own quality but the quality of its predecessors. If the cumulative quality of a certain group is lower than an established threshold then rework is needed. The model's objective is to select execution modes for tasks to minimize rework costs and direct costs that decrease linearly with time.

In this research we extend the work of Liberatore and Pollack-Johnson (2013) to include the effect of time and cost uncertainty on task quality and the optimal solution to the continuous version of the TCQTP. This extension results in the STCQTP, which provides optimal hedging against these uncertainties.

\section{Maximizing Project Quality: The Deterministic Case}

Liberatore and Pollack-Johnson (2013) introduced a framework for finding cost (budgeted expenditure) and time (planned) values to maximize overall project quality using continuous quality functions that have two basic properties:

- Holding time constant, quality $(q)$ is an increasing function of cost.

- Holding cost constant, quality is an increasing function of time.

Quality is lowest at the corner of the domain with the smallest values of time and cost and highest in the opposite corner (the highest values of time and cost). For a fixed quality, we obtain a standard time/cost tradeoff curve that is decreasing and convex. Thus, to maintain the same level of quality, to reduce the time, one must pay increasingly more money per unit, such as in standard project activity crashing (Brucker et al. 1999). This suggests a basic hill shape rising out of a plain, although one would only be interested in a one-quarter wedge of the hill (as in Figure 1).

The quality function is normalized so that the maximum time $\left(\mu_{t}\right)$ and cost $\left(\mu_{c}\right)$ values considered reasonable correspond to a constant indicating the maximum quality level possible. The "standard deviation" parameters ( $\sigma_{t}$ and $\left.\sigma_{c}\right)$ give a measure of how slowly the quality drops from the top of the hill compared to the maximum values for time and cost, respectively.

These authors used the following modified bivariate normal quality function, where $K$ is the maximum quality level

$$
Q(t, c)=K e^{-\left[\left(\frac{t-\mu_{t}}{\sigma_{t}}\right)^{2}+\left(\frac{c-\mu_{c}}{\sigma_{c}}\right)^{2}\right]}
$$

A typical shape of the quality function is shown as Figure 1. Note that the maximum quality occurs at $\mu_{t}$ and $\mu_{c}$. 


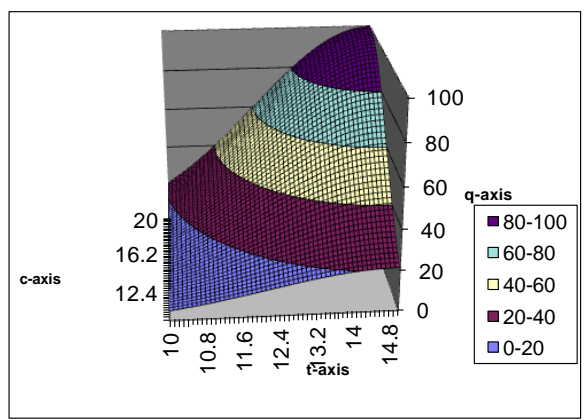

Figure 1 Bivariate Normal Quality Function

This quality function is incorporated into the following nonlinear programming model whose objective is to maximize project quality, expressed as the minimum quality of the individual tasks, so that the quality of the project overall is defined by the quality of the weakest link:

Maximize $Q_{\min }$

Subject to

$$
Q_{\min } \leq q_{i}, i=1,2, \ldots N
$$

$q_{i}=Q_{i}\left(t_{i}, c_{i}\right)=K \cdot e^{-\left(\frac{t_{i}-\mu_{t_{i}}}{\sigma_{t_{i}}}\right)^{2}-\left(\frac{c_{i}-\mu_{c_{i}}}{\sigma_{c_{i}}}\right)^{2}}, i=1,2, \ldots N$

$\sum_{i=1}^{N} c_{i} \leq C_{U B}$

$s_{0}=0$

$s_{k} \geq s_{i}+t_{i} \quad \forall i=0, \ldots, N, \forall k \in S_{i}$

$s_{N+1} \leq T_{U B}$

$s_{i} \geq 0 \quad \forall i=1, \ldots, N+1$

$t_{i} \geq t_{\min _{i}}, i=1,2, \ldots, N$

$c_{i} \geq c_{\min _{i}}, i=1,2, \ldots, N$ 


$$
\begin{aligned}
& t_{i} \leq \mu_{t_{i}}, i=1,2, \ldots, N \\
& c_{i} \leq \mu_{c_{i}}, i=1,2, \ldots, N \\
& q_{i}, t_{i}, c_{i} \geq 0, i=1,2, \ldots, N
\end{aligned}
$$

Where

$t_{i}=$ the duration of activity $i$, for $i=1, \ldots, N$

$c_{i}=$ the cost of activity $i$, for $i=1, \ldots, N$

$q_{i}=$ the quality of activity $i$, for $i=1, \ldots, N$

$Q_{\min }=$ a decision variable used to obtain the minimum quality over all the activities

$S_{i}=$ the set of activities that are immediate successors of activity $i$, for $i=0, \ldots, N$

$T_{U B}=$ upper bound on the total project time

$C_{U B}=$ upper bound on the total project cost

$s_{i}=$ the scheduled start time for activity $i$, for $i=0, \ldots, N+1$

$t_{\min _{i}}=$ lower bound on the duration of activity $i$, for $i=1, \ldots, N$

$c_{\text {min }_{i}}=$ lower bound on the cost of activity $i$, for $i=1, \ldots, N$

Equations (1) - (3) use the nonlinear continuous quality function to define the quality of each activity and find the minimum quality of all the activities. Equation (4) is the budget constraint, and equations (5) - (8) make sure the activity start times reflect the precedence relations. Equations (9) - (13) ensure the bounds for time and cost of each activity are reasonable, such as by ensuring the quality function stays increasing in both variables.

This model can be modified slightly to produce time-cost trade-off curves for different quality levels. Equation (1) can be changed to

Minimize $C=\sum c_{j}$

and equation (4) can be replaced with

$Q_{\min } \geq Q_{0}$

For a given quality level, $Q_{0}$, one can then specify an upper bound on the total project time, $T_{U B}$, and find the minimum cost $(C)$ possible to achieve at least a $Q_{0}$ level of quality within that amount of time. Specifying different $T_{U B}$ values will then give a time-cost trade-off curve for that level of quality. Varying the values of $Q_{0}$ will then provide quality level curves for the overall project quality, so a project manager can decide which combination of project deadline, budget, and quality makes the most sense for their particular problem.

\section{The Effect of Uncertainty: The Translator Example}

In this section we show how uncertainty related to task time can affect the optimal level of project quality achieved using a translator project example, leading to our 
general formulation of the STCQTP. This discussion is motivated by Liberatore and Pollack-Johnson (2013), who gave an example where the original deterministic model above could be used during a project if a certain task took much longer than expected, such as a project task that is delayed due to inclement weather. A second round of optimization in the middle of the project could then be done to adjust for the unexpected circumstances (with new estimates of the parameters of the quality function of at least the time parameter(s) of the delayed task), to try to minimize the damage to time, cost, and quality for the project. Thus, this delay could be modeled as a deterministic change in the time parameter/s. Traditional analysis does not have a way to incorporate quality as a variable in this process, but the Liberatore and Pollack-Johnson (2013) approach does.

This raises the question of how an analog of such an analysis might be performed in advance, with a probability distribution related to the uncertainty in the time and/or cost values. If we could represent this stochastic possibility using existing data or even subjective estimation to obtain a probability distribution, we could extend the approach by maximizing the expected overall quality of the project. In this way, our proposed approach can then lead to a method for optimally hedging against these uncertainties.

As an example, let us consider the translator problem from Liberatore and PollackJohnson (2013), where two translators are each working on a large translation, and the project manager at the translation agency has estimated quality functions for each of them. The data from this work is summarized in Table 1. In the original real-life example, the activities were in parallel, since the translators could work on the two pieces at the same time. The consequences of uncertainty will be more interesting and illustrative if we use the same quality functions derived in that article, but consider the activities to be in series. As a result, a time delay in the earlier activity will have a bigger effect on the second activity and the project as a whole, so we have made that modification here.

Table 1 Translator Project: Time, Cost and Quality Estimates

\begin{tabular}{|c|c|c|c|c|c|}
\hline \multicolumn{3}{|c|}{ Translator 1 } & \multicolumn{3}{c|}{ Translator 2 } \\
\hline $\begin{array}{c}\text { Deadline } \\
(\text { days })\end{array}$ & $\begin{array}{c}\text { Cost } \\
(\$)\end{array}$ & $\begin{array}{c}\text { Quality } \\
(0-44)\end{array}$ & $\begin{array}{c}\text { Deadline } \\
(\text { days })\end{array}$ & $\begin{array}{c}\text { Cost } \\
(\$)\end{array}$ & $\begin{array}{c}\text { Quality } \\
(0-44)\end{array}$ \\
\hline$t$ & $c$ & $q$ & $t$ & $c$ & $q$ \\
\hline 4 & 1435 & 41 & 4 & 653 & 32 \\
\hline 3 & 1435 & 35 & 3.75 & 653 & 26 \\
\hline 3 & 1750 & 41 & 3.75 & 800 & 32 \\
\hline 3 & 2150 & 43 & 3.75 & 975 & 33 \\
\hline 2.5 & 2150 & 37 & 3.5 & 975 & 26 \\
\hline 2 & 2150 & 33 & & & \\
\hline
\end{tabular}

From equations (11) and (12), we see that the $\mu$ parameters need to be greater than or equal to the corresponding $\mathrm{t}$ and $c$ data values. Thus, to fit a model to this data, we need to impose that condition on the two $\mu$ parameters, so we find the model 
parameters satisfying those constraints that minimize the sum of the squared residuals. Using the data points from Table 1, the four quality function parameter values were estimated using the constrained nonlinear regression analysis function within IBM SPSS Statistics version 24 (IBM Corp. 2016). The values of $K$ that fit best for tasks 1 and 2 were 50 and 44, respectively. The estimated values are summarized in Table 2.

Table 2 Translator Project: Quality Function Parameter Estimates for Both Tasks

\begin{tabular}{|c|c|c|}
\hline Parameter & Task 1 & Task 2 \\
\hline$\mu_{t_{i}}$ & 4.509 & 4.377 \\
\hline$\sigma_{t_{i}}$ & 3.822 & 1.201 \\
\hline$\mu_{c_{i}}$ & 2150.000 & 975.000 \\
\hline$\sigma_{c_{i}}$ & 1655.086 & 671.098 \\
\hline
\end{tabular}

Let us now consider the case where the situation for task 1 changes, and the project manager realizes this before task 1 is completed, thus requiring a reevaluation of the planned times and costs. For example, suppose the translator working on task 1 has a family emergency that just shifts the quality function that would be associated with the actual (clock/calendar) time that the task takes. This shift, a deterministic constant additive factor $(\Delta t)$, represents the time that they were out of commission and not able to work on the translation. This would be a horizontal shift to the right of the quality function in the time dimension, so the adjusted quality function is

$Q_{1}^{\mathrm{A}}\left(t_{1}, c_{1}\right)=Q_{1}\left(t_{1}-\Delta t, c_{1}\right)$.

If we define $Q_{1}{ }^{\mathrm{B}}\left(t_{1}, c_{1}\right)$ to be the same as the original quality function for task 1 , $Q_{1}\left(t_{1}, c_{1}\right)$, but with the $\mu_{t_{1}}$ parameter replaced by $\left(\mu_{t_{1}}+\Delta t\right)$, we see that

$\begin{aligned} Q_{1}^{\mathrm{B}}\left(t_{1}, c_{1}\right) & =K e^{-\left(\frac{t_{1}-\left(\mu_{t_{1}}+\Delta t\right)}{\sigma_{t_{1}}}\right)^{2}-\left(\frac{c_{1}-\mu_{c_{1}}}{\sigma_{t_{1}}}\right)^{2}}=K e^{-\left(\frac{\left(t_{1}-\Delta t\right)-\mu_{t_{1}}}{\sigma_{t_{1}}}\right)^{2}-\left(\frac{c_{1}-\mu_{c_{1}}}{\sigma_{t_{1}}}\right)^{2}} \\ & =Q_{1}\left(t_{1}-\Delta t, c_{1}\right)=Q_{1}^{\mathrm{A}}\left(t_{1}, c_{1}\right) .\end{aligned}$

In other words, we see that $Q_{1}{ }^{\mathrm{A}}\left(t_{1}, c_{1}\right)=Q_{1}\left(t_{1}-\Delta t, c_{1}\right)$ is mathematically equivalent to $Q_{1}^{\mathrm{B}}\left(t_{1}, c_{1}\right)$. Thus, we can think of this unexpected monkey wrench, $\Delta t$, as a change to the parameter $\mu_{t_{1}}$. The original shape of the quality function is still the same; it is just shifted.

For a given constant value of $\Delta t$, this is just another variant of the original deterministic maximization of the overall quality, so can be solved as before. Thinking of the context of the time delay, if you did not take the time to calculate an exact optimal approach, you might consider two simple extreme heuristic strategies: (1) still take the original assigned time $t_{1}$ for task 1 , but sustain a large drop in the quality of task 1 because of the shift in the quality function (in effect, taking less time to really do the task than originally planned for), or (2) add the $\Delta t$ to the scheduled time for task 1 (accommodate the delay fully, so add the total time out of commission to the planned time allocated to task 1), and let task 2 suffer (its planned 
time will need to decrease by the same amount, in order to meet the time deadline, which will lower its quality). In real life, option (2) is probably the most common, leading to a delay of the whole project or a major quality (or cost) disaster in rushing task 2 to get done in time before the hard deadline. These two extreme heuristics are useful benchmarks to compare the optimal solution to, in order to see the potential improvement from using the optimization compared to the simplistic extreme heuristics.

We began with the baseline case $(\Delta t=0)$ where we assume a constraint of $\$ 2400$ for the total cost (as in the original example), and 6.2 days for the whole project. Using the Lingo Global Solver (LINDO Systems 2019) we obtained a solution in 0.60 seconds with an optimal project quality of 33.0 as shown in Table. 3 . This and all subsequent problems were run on a Dell Latitude E6440 with an Intel Core i5 CPU at $270 \mathrm{Ghz}$.

Table 3 Translator Project: Optimal Deterministic Solution $\left(Q^{*}=33.0\right)$

\begin{tabular}{|c|c|c|c|}
\hline Task $(\boldsymbol{i})$ & Time $\left(\boldsymbol{t}_{\boldsymbol{i}}\right.$, in days $)$ & Cost $\left(\boldsymbol{c}_{\boldsymbol{i}}\right.$, in $\left.\mathbf{\text { }}\right)$ & Quality $\left(\boldsymbol{q}_{\boldsymbol{i}}\right.$, on 0-44 scale $)$ \\
\hline 1 & 2.35 & 1634.10 & 33.0 \\
\hline 2 & 3.85 & 765.90 & 33.0 \\
\hline
\end{tabular}

In the translator example we then looked at the solutions for different values of $\Delta t$, and compared them to the simplistic extreme heuristics: Heuristic 1 - "stick with the original schedule for task 1" and Heuristic 2 - "fully accommodate the delay in the scheduled time for task 1". Figure 2 summarizes the Excel Solver results, which show that, even with only a $\Delta t$ of 0.5 (half a day delay), the overall quality for the optimal solution is $5 \%$ better than Heuristic 1, and about $70 \%$ better than Heuristic 2 .

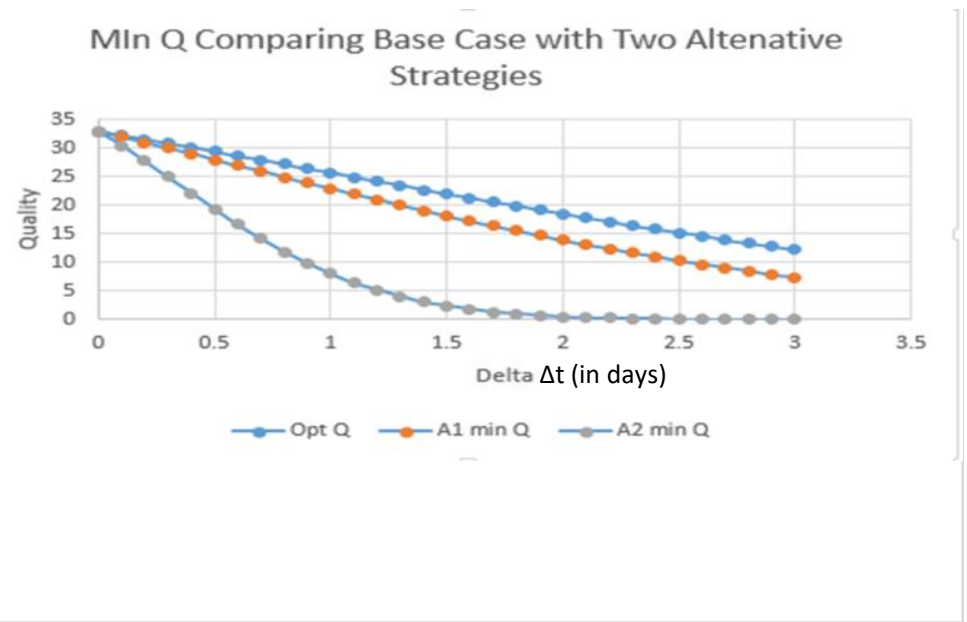

Figure 2 Translator Project: Sensitivity of Min Q to Increases in At (Ai is Heuristic i)

Now we consider the case where, rather than the $\Delta t$ for task 1 being a known, constant amount, it is assigned a probability distribution. We saw above that the time 
delay of $\Delta t$ is equivalent to a shift in the $\mu_{t_{1}}$ parameter of the quality function by $\Delta t$. Thus, the deterministic problem is generalized to incorporate stochastic parameters.

Specifically, by way of illustration, we considered the case where $\Delta t$ follows a triangular probability distribution with a minimum of 0 , a mode of 0 , and a maximum of 2 . The pdf is given by

$f(t)=\left\{\begin{array}{c}1-\frac{1}{2} t, \text { for } 0 \leq t \leq 2 \\ 0, \text { otherwise }\end{array}\right.$

In the general case, all of the quality function parameters could be stochastic, leading to the STCQTP:

$\operatorname{Max} \mathrm{E}\left[\min _{i}\left\{\left(\tilde{Q}_{i}\left(t_{i}, c_{i}\right)\right\}\right]\right.$

Subject to

$\tilde{Q}_{i}\left(t_{i}, c_{i}\right)=K e^{-\left(\frac{t_{i}-\widetilde{\mu}_{t_{i}}}{\widetilde{\sigma}_{t_{i}}}\right)^{2}-\left(\frac{c_{i}-\widetilde{\mu}_{c_{i}}}{\tilde{\sigma}_{c_{i}}}\right)^{2}} i=1,2, \ldots, N$

and equations (4)-(13),

where at least one of the parameters of the quality function is stochastic, indicated by the tilde notation.

Returning to our immediate translator problem,

$\tilde{Q}_{1}\left(t_{1}, c_{1}\right)=Q_{1}\left(t_{1}+\widetilde{\Delta t}, c_{1}\right)$,

where $\widetilde{\Delta t}$ has the pdf given in (16). Since $\widetilde{Q}_{2}\left(t_{2}, c_{2}\right)$ is assumed to be deterministic,

$\tilde{Q}_{2}\left(t_{2}, c_{2}\right)=Q_{2}\left(t_{2}, c_{2}\right)$,

as in equation (3) of the deterministic problem. Therefore, our objective equation (17) becomes

$\operatorname{Max} \mathrm{E}\left[\min \left\{\tilde{Q}_{1}\left(t_{1}, c_{1}\right), Q_{2}\left(t_{2}, c_{2}\right)\right\}\right]$

where

$$
\begin{array}{r}
\mathrm{E}\left[\min \left\{\tilde{Q}_{1}\left(t_{1}, c_{1}\right), Q_{2}\left(t_{2}, c_{2}\right)\right\}\right]=Q_{2}\left(t_{2}, c_{2}\right) \cdot \operatorname{Prob}\left\{\tilde{Q}_{1}\left(t_{1}, c_{1}\right)>Q_{2}\left(t_{2}, c_{2}\right)\right\}+ \\
E\left[\tilde{Q}_{1}\left(t_{1}, c_{1}\right) \mid \tilde{Q}_{1}\left(t_{1}, c_{1}\right) \leq Q_{2}\left(t_{2}, c_{2}\right)\right] \cdot \operatorname{Prob}\left\{\tilde{Q}_{1}\left(t_{1}, c_{1}\right) \leq Q_{2}\left(t_{2}, c_{2}\right)\right\}
\end{array}
$$

The stochastic programming feature of LINGO (LINDO Systems 2019) was used to solve this problem. The stochastic program is a multistage model which, in our case, means that decisions on task time and cost are made at stage zero and uncertainty related to the quality function parameters are resolved in stage one. The 
solution procedure generated 100 samples of random values of $\Delta t$, forming 100 scenarios. The global optimal value for expected project quality is the average of the minimum quality values resulting when the optimal task time and cost solutions are applied to the 100 sample cases.

The global optimal solution is shown in Table 4. The global optimum was identified quickly, but convergence was slow. Determining the local optimum requires much less computational time $(0.29$ seconds $)$ and also gives the global optimal solution. In order to significantly reduce runtimes for finding the global optimum, we took the natural logarithm of the quality function, affecting equations (1) - (3) of the model formulation. The converted problem is a stochastic quadratic program, rather than a stochastic general nonlinear program, allowing the more efficient quadratic solver to be used. The revised formulation obtains the same global optimal solution, but in much less computational time ( 0.42 seconds).

Notice that comparing the stochastic results in Table 4 with the deterministic results in Table 3 we see that $t_{1}$ has been increased by a small amount and that $c_{1}$ has decreased slightly, but the overall quality has decreased by over 6 points, as would be expected, since the time delay will necessarily have to lower the overall project quality.

The optimal expected value solution was determined by assuming that the uncertain parameters are each fixed at their expected values. This expected value solution was then applied to the stochastic problem, yielding an expected quality of 26.2. The expected value of the optimal solution to the stochastic problem is 26.7 (Table 4), and the increase of 0.5 represents the improvement gained from the optimal hedge against the uncertainty (vs the expected value solution).

Table 4 Translator Project: Optimal Stochastic Solution $\left(Q^{*}=26.7\right)$

\begin{tabular}{|c|c|c|c|}
\hline Task $(\boldsymbol{i})$ & Time $\left(\boldsymbol{t}_{\boldsymbol{i}}\right.$, in days) & Cost $\left(\boldsymbol{c}_{\boldsymbol{i}}\right.$, in $\left.\mathbf{\$}\right)$ & Expected Quality (on 0-44 scale) \\
\hline 1 & 2.46 & 1617.87 & 27.3 \\
\hline 2 & 3.74 & 782.13 & 30.5 \\
\hline
\end{tabular}

Note that the minimum of the expected task quality values, 27.3 (see Table 4), is larger than the optimal expected quality of 26.7 ; that is:

$\min \left\{E\left(\tilde{Q}_{i}\left(t_{i}, c_{i}\right)\right)\right\}>E\left(\min \left\{\tilde{Q}_{i}\left(t_{i}, c_{i}\right)\right\}\right)$

In this case, since $Q_{2}$ is deterministic at the optimal solution, its value is always 30.5. However, since $Q_{1}$ is stochastic, it turns out that there is a positive probability its value can be more than 30.5 , and these values are included in the expected value of $Q_{1}$, which in this case makes its individual expected quality higher than the overall expected quality.

From this example, we see that time uncertainty can be modeled by making the quality function parameters stochastic. We see that the most obvious heuristics can perform poorly compared to the optimal solution in the presence of such unexpected changes. Our examples show that the optimal solution to the stochastic problem can 
differ significantly from the deterministic and expected value solutions and yields an optimal hedging strategy against the uncertainty.

A possible extension of this modeling strategy would be to allow for a correlation cross product term in the quality function (analogous to a general bivariate normal density function). To use this approach, we added the term

$$
-2 \rho\left(\frac{t_{i}-\mu_{t_{i}}}{\sigma_{t_{i}}}\right)\left(\frac{c_{i}-\mu_{c_{i}}}{\sigma_{c_{i}}}\right)
$$

to the exponent of the quality function. We then applied the same least squares approach to estimating all of the parameters of the quality function for Task 1 as before, but now with 5 parameters instead of 4 . A local optimal solution was found quickly, and the original 4 parameters all agreed with the values given earlier, to 3 or 4 significant figures, with an estimated $\rho$ value of 0.00916 , not significantly different from 0 . For other applications, correlations could be significant, and this extension could be utilized.

\section{Software Development Project}

The software development project discussed here was originally presented in Liberatore and Pollack-Johnson (2013). This project involves gathering the customer requirements, then developing a quote for the project cost. If the quote is accepted, staff are allocated, there is an initial team meeting, and the main database and software configuration and testing are pursued in parallel (as well some tasks that are outsourced). These pieces are integrated and finalized, and there is a final task of analyst testing before the software is sent to the client for their testing. Task descriptions and precedence are found in Table 5. For tasks 1, 4, 5, 6 and 7, quality was measured for different combinations of time and cost based on the expected impact of possible rework cycles. No rework cycles were possible for tasks 2 and 3 so their quality values are set at 100 and their task times and costs are set. Each of tasks $1,4,5,6$ and 7 has four estimated quality function parameters: $\mu_{t_{i}}, \sigma_{t_{i}}, \mu_{c_{i}}$, and $\sigma_{c_{i}}$. These values were estimated, again using the constrained nonlinear regression analysis function within IBM SPSS Statistics version 24 (IBM Corp. 2016). If the estimated standard error (SE) was greater than $25 \%$ of the estimated mean, the SE was replaced by $0.25^{*}$ (estimated mean). This was done because in some cases the estimated standard error of a parameter was quite large, possibly due to the limited data available for a particular task. By limiting the standard error to $.25^{*}$ estimated parameter mean, the effect of a parameter's variability will not have undue influence on the optimal solution. Allowing larger variation would produce simulated values that would not make sense in the real world context. The estimated mean values, along with their adjusted SEs, are summarized in Table 5. Note that $K=100$ for the quality functions for tasks $1,4,5,6$ and 7, and that tasks 0 and 8 are dummies. 
Table 5 Software Project: Precedence and Time and Cost Parameter Estimation

\begin{tabular}{|c|c|c|c|c|c|c|c|c|c|c|}
\hline \multirow[t]{2}{*}{ Task $(i)$} & \multirow[t]{2}{*}{ Description } & \multirow[t]{2}{*}{ Pred. } & \multicolumn{2}{|c|}{$\mu_{t_{i}}$} & \multicolumn{2}{|c|}{$\sigma_{t_{i}}$} & \multicolumn{2}{|c|}{$\mu_{c_{i}}$} & \multicolumn{2}{|c|}{$\sigma_{c_{i}}$} \\
\hline & & & Est. & $\begin{array}{l}\text { Adj. } \\
\text { SE }\end{array}$ & Est. & $\begin{array}{l}\text { Adj. } \\
\text { SE }\end{array}$ & Est. & $\begin{array}{l}\text { Adj. } \\
\text { SE }\end{array}$ & Est. & $\begin{array}{l}\text { Adj. } \\
\text { SE }\end{array}$ \\
\hline 0 & Dummy & -- & 0 & & 0 & & 0 & & 0 & \\
\hline 1 & $\begin{array}{l}\text { Requirements \& } \\
\text { Review }\end{array}$ & 0 & 4.33 & 0.23 & 10.42 & 0.88 & 204.00 & $51.00^{1}$ & 116.24 & $29.06^{1}$ \\
\hline 2 & Setup & 1 & 4.00 & & 0 & & 1045.00 & & 0 & \\
\hline 3 & $\begin{array}{l}\text { Vendor } \\
\text { Configuration \& }\end{array}$ & 2 & 1.00 & & 0 & & 1000.00 & & 0 & \\
\hline 4 & \begin{tabular}{|l} 
Standard \\
Configuration \&
\end{tabular} & 2 & 12.00 & 2.76 & 12.82 & $3.21^{1}$ & 525.00 & $131.25^{1}$ & 520.60 & $130.15^{1}$ \\
\hline 5 & $\begin{array}{l}\text { Data Base } \\
\text { Configuration \& }\end{array}$ & 2 & 12.55 & 2.13 & 11.16 & $2.79^{1}$ & 5493.10 & 588.27 & 4004.92 & 832.22 \\
\hline 6 & $\begin{array}{l}\text { Development, } \\
\text { Integration \& }\end{array}$ & $3,4,5$ & 1.54 & $0.39^{1}$ & 1.88 & $0.47^{1}$ & 56.16 & 11.95 & 52.06 & $13.02^{1}$ \\
\hline 7 & $\begin{array}{l}\text { Quality Assurance } \\
\text { Testing }\end{array}$ & 6 & 1.00 & 0.16 & 1.28 & $0.32^{1}$ & 417.07 & $104.27^{1}$ & 1437.77 & $359.44^{1}$ \\
\hline 8 & Dummy & 7 & 0 & & 0 & & 0 & & 0 & \\
\hline
\end{tabular}

${ }^{1} \mathrm{SE}$ was replaced by $.25 * \mu$

Using the estimated values for $\mu_{t_{i}}, \sigma_{t_{i}}, \mu_{c_{i}}$, and $\sigma_{c_{i}}$ for the task quality functions and equations (1) - (13), the optimal software project quality for the deterministic version of the time-cost-quality problem were found using LINGO's global nonlinear solver (LINDO Systems 2019). Here we assume $T_{U B}=18$ and $C_{U B}=$ $\$ 7,000$. The results are given on the project network shown as Figure 3. For tasks 1 , 4, 5, 6 and 7 the optimal solution led to each of these tasks having a quality value of 89.2 , which is also the optimal objective value.

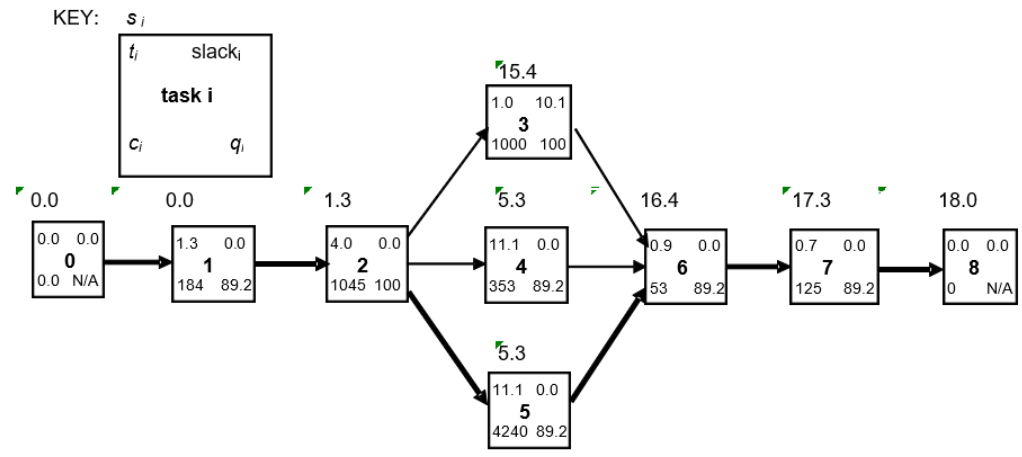

Figure 3 Software Project: Optimal Deterministic Solution (Optimal Quality = 89.2) 
Using the deterministic solution as a baseline, we next consider the stochastic version of the time-cost-quality problem using equations (4) - (13), (17), and (18). Triangular probability distributions were used to represent uncertainty for the quality function time and cost parameters for tasks 1, 4, 5, 6 and 7. The estimated parameter value and its adjusted SE (Table 5) were used to specify the triangular distribution parameters as follows: the most likely (ML) value of the triangular distribution is the estimated value of the parameter, and the lower bound (LB) and upper bound (UB) values are one adjusted SE below and above the estimated parameter value, respectively. The time and cost triangular distributions can be found in Tables 6 and 7 , respectively.

Table 6 Software Project: Triangular Distributions for Time Parameters

\begin{tabular}{|c|c|c|c|c|c|c|}
\hline & \multicolumn{3}{|c|}{$\boldsymbol{\mu}_{\mathbf{t}_{\mathbf{i}}}$} & \multicolumn{3}{c|}{$\sigma_{\mathbf{t}_{\mathbf{i}}}$} \\
\hline Task(i) & LB & ML & UB & LB & ML & UB \\
\hline 1 & 4.10 & 4.33 & 4.56 & 9.55 & 10.42 & 11.30 \\
\hline 2 & & 4.00 & & & 0.00 & \\
\hline 3 & & 1.00 & & & 0.00 & \\
\hline 4 & 9.24 & 12.00 & 14.77 & 9.61 & 12.82 & 16.02 \\
\hline 5 & 10.42 & 12.55 & 14.68 & 8.38 & 11.16 & 13.94 \\
\hline 6 & 0.92 & 1.54 & 2.17 & 1.41 & 1.88 & 2.36 \\
\hline 7 & 0.84 & 1.00 & 1.16 & 0.96 & 1.28 & 1.60 \\
\hline
\end{tabular}

Table 7 Software Project: Triangular Distributions for Cost Parameters

\begin{tabular}{|c|c|c|c|c|c|c|}
\hline & \multicolumn{3}{|c|}{$\boldsymbol{\mu}_{\boldsymbol{c}_{\boldsymbol{i}}}$} & \multicolumn{3}{c|}{$\sigma_{\boldsymbol{c}_{\boldsymbol{i}}}$} \\
\hline Task $(i)$ & LB & ML & UB & LB & ML & UB \\
\hline 1 & 153.00 & 204.00 & 255.00 & 87.18 & 116.24 & 145.30 \\
\hline 2 & & 1045.00 & & & 0.00 & \\
\hline 3 & & 1000.00 & & & 0.00 & \\
\hline 4 & 393.15 & 525.00 & 656.25 & 390.44 & 520.65 & 650.75 \\
\hline 5 & 4904.83 & 5493.10 & 6081.37 & 3172.70 & 4004.92 & 4837.14 \\
\hline 6 & 44.21 & 56.16 & 68.11 & 39.04 & 52.06 & 65.07 \\
\hline 7 & 312.80 & 417.07 & 521.34 & 1070.88 & 1437.77 & 1794.71 \\
\hline
\end{tabular}

Using the same stochastic modeling approach as discussed for the translator problem (see section 4), the global optimal solution for the stochastic software problem using triangular probability distributions for the uncertain time and cost was found and is shown on the project network in Figure 4. As in the translator problem, the global optimum was found early, but convergence was slow (108.94 seconds). A local optimal solution that is the global optimum is found in only 2.07 seconds. Taking natural logs of the quality function as for the translator problem also yields the global optimum in only 1.54 seconds. Based on the performance of the natural 
$\log$ formulation for the translator and software problems, we believe that it is the preferred solution approach.

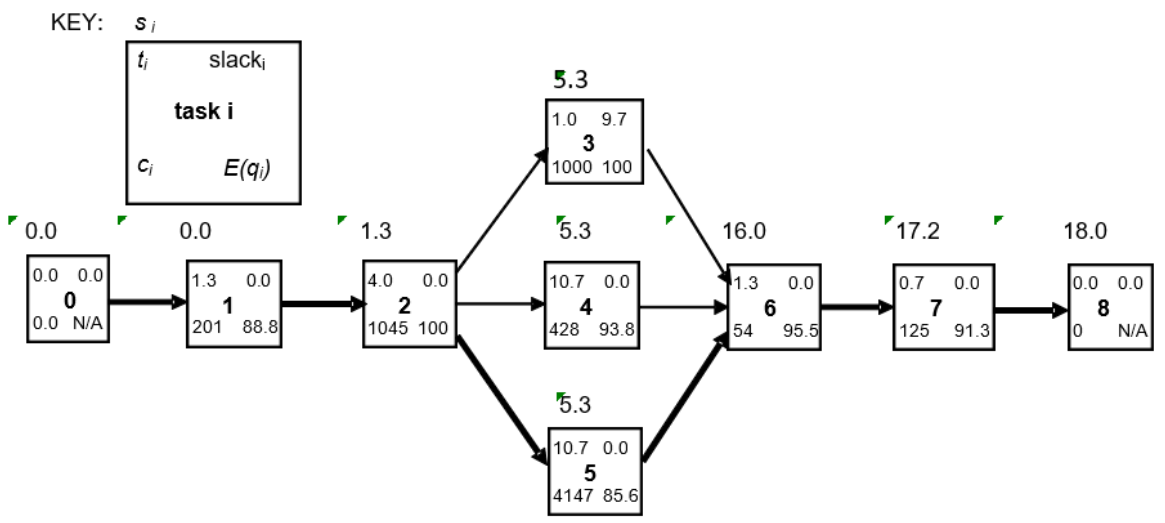

Figure 4 Software Project: Optimal Stochastic Solution (optimal expected quality = 84.0)

Unlike the optimal deterministic solution, the expected quality values for tasks 1 , 4, 5, 6, and 7 are not equal. The stochastic optimal expected quality equals 84.0. As in the translator problem, this value is computed as the average of the minimum of the task quality values based on the optimal $t_{i}$ and $c_{i}$ values for each of the 100 samples generated during the stochastic optimization process. Note that, as was true for the translator problem, the minimum of the expected task quality values, $\min \left\{E\left(\tilde{Q}_{i}\left(t_{i}, c_{i}\right)\right)\right\}=85.6$, (see Figure 4 ) is not the optimal expected quality of 84.0 (see equation (19)).

The cumulative frequency distribution of project quality (based on the 100 samples) is shown as Figure 5. There is some downside risk, with a $20 \%$ chance that project quality will be below 80 . However, there is a $57 \%$ chance that project quality will be greater than the optimal expected value (84.0).

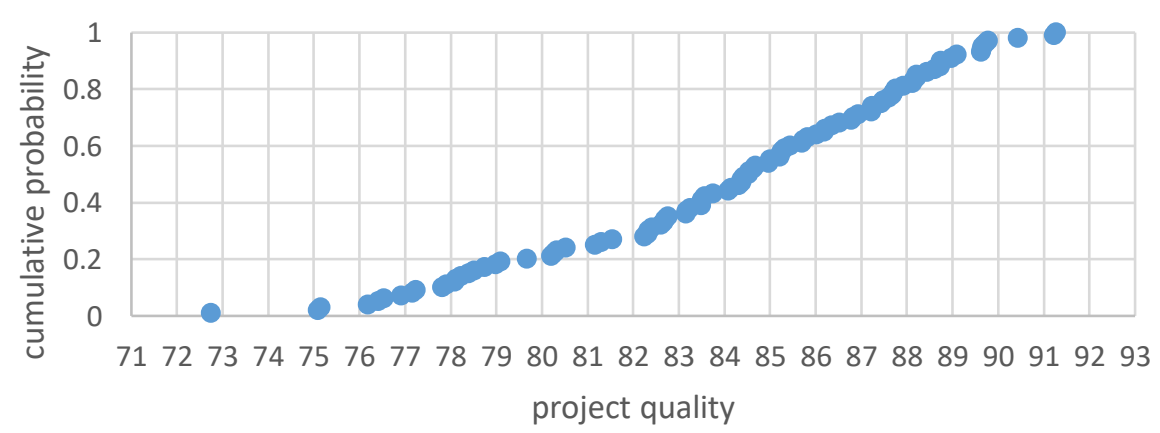

Figure 5 Cumulative Probability Distribution of Software Project Quality 
The results show that the deterministic solution (the same as the expected value solution, in this case) overestimates project quality by $100 *(89.2-84.0) / 84.0=6.2 \%$. If the optimal deterministic task times and costs are set in the stochastic model, the results lead to a project quality of 76.8 or a decrease of $100 *(84.2-76.8) / 84.2=8.8 \%$. Therefore, the effect of uncertainty on the optimal solution is significant and using the deterministic policies can lead to lower project quality. Once again, the solution to the stochastic problem yields an optimal hedging strategy against the time and cost uncertainties.

\subsection{Sensitivity to the Form of the Probability Distribution}

To explore the sensitivity of the global optimal solution to the assumption about the probability distribution of the quality function parameters, we also solved the software stochastic optimization model using the normal and uniform distributions. For the normal distributions the mean values were set as the ML values from the triangular distributions (Tables 6 and 7), while the standard deviations were set to one-third of the adjusted SE values from Table 5, so that the normal means $+/-$ three standard deviations match the UB and LB values, respectively, in the triangular distributions. The normal distribution parameters are found in Table 8. For the uniform distributions we set the minimum and maximum values to the triangular LB and UB values, respectively. There the range of possible values for each probability distribution are comparable. All problems were run using the natural log formulation and solved in under two seconds, with the results for the triangular, normal and uniform distributions given in Table 9. The triangular and normal results are similar, while the uniform results are generally lower, especially for the objective value. This could be due to the higher kurtosis of the uniform distribution, but is most likely primarily due to its higher variance, since the higher variability makes an effective response harder on average. The results show some sensitivity to the distributional assumption concerning the model's parameters.

Table 8: Normal Distribution Parameters for Time and Cost Means and Standard Deviations for the Software Problem

\begin{tabular}{|c|l|c|c|c|c|c|c|c|c|c|}
\hline Task(i) & \multicolumn{1}{|c|}{ Description } & Pred. & \multicolumn{1}{|c|}{$\boldsymbol{\mu}_{\boldsymbol{t}_{\boldsymbol{i}}}$} & \multicolumn{2}{|c|}{$\sigma_{\boldsymbol{t}_{\boldsymbol{i}}}$} & \multicolumn{2}{|c|}{$\boldsymbol{\mu}_{\boldsymbol{c}_{\boldsymbol{i}}}$} & \multicolumn{2}{|c|}{$\sigma_{\boldsymbol{c}_{\boldsymbol{i}}}$} \\
\hline & & & mean & $\begin{array}{l}\text { Std. } \\
\text { dev. }\end{array}$ & mean & Std.dev. & mean & $\begin{array}{c}\text { Std. } \\
\text { dev. }\end{array}$ & mean & $\begin{array}{c}\text { Std. } \\
\text { dev. }\end{array}$ \\
\hline 0 & Dummy & 0 & & 0 & & 0 & & 0 & \\
\hline 1 & $\begin{array}{l}\text { Requirements \& } \\
\text { Review }\end{array}$ & 0 & 4.33 & 0.08 & 10.42 & 0.29 & 204.00 & 16.98 & 116.24 & 9.68 \\
\hline 2 & Setup & 4.00 & & 0 & & 1045.00 & & 0 & \\
\hline 3 & $\begin{array}{l}\text { Vendor } \\
\text { Configuration \& }\end{array}$ & 2 & 1.00 & & 0 & & 1000.00 & & 0 & \\
\hline 4 & $\begin{array}{l}\text { Standard } \\
\text { Configuration \& }\end{array}$ & 2 & 12.00 & 0.92 & 12.82 & 1.07 & 525.00 & 43.71 & 520.60 & 43.34 \\
\hline 5 & $\begin{array}{l}\text { Data Base } \\
\text { Configuration \& }\end{array}$ & 2 & 12.55 & 0.71 & 11.16 & 0.93 & 5493.10 & 195.89 & 4004.92 & 277.13 \\
\hline
\end{tabular}




\begin{tabular}{|c|l|c|c|c|c|c|c|c|c|c|}
\hline 6 & $\begin{array}{l}\text { Development, } \\
\text { Integration \& }\end{array}$ & $\begin{array}{c}3,4, \\
5\end{array}$ & 1.54 & 0.21 & 1.88 & 0.16 & 56.16 & 3.98 & 52.06 & 4.34 \\
\hline 7 & $\begin{array}{l}\text { Quality Assurance } \\
\text { Testing }\end{array}$ & 6 & 1.00 & 0.05 & 1.28 & 0.11 & 417.07 & 34.72 & 1437.77 & 119.69 \\
\hline 8 & Dummy & 7 & 0 & & 0 & & 0 & & 0 & \\
\hline
\end{tabular}

Table 9 Software Problem Solution Results by Probability Distribution

\begin{tabular}{|l|c|c|c|}
\hline & Triangular & Normal & Uniform \\
\hline $\mathrm{E}\left(\mathrm{Q}_{1}\right)$ & 88.8 & 89.7 & 85.8 \\
\hline $\mathrm{Q}_{2}$ & 100 & 100 & 100 \\
\hline $\mathrm{Q}_{3}$ & 100 & 100 & 100 \\
\hline $\mathrm{E}\left(\mathrm{Q}_{4}\right)$ & 93.8 & 93.5 & 93.3 \\
\hline $\mathrm{E}\left(\mathrm{Q}_{5}\right)$ & 85.6 & 86.4 & 83.5 \\
\hline $\mathrm{E}\left(\mathrm{Q}_{6}\right)$ & 95.5 & 95.1 & 94.1 \\
\hline $\mathrm{E}\left(\mathrm{Q}_{7}\right)$ & 91.3 & 91.2 & 91.8 \\
\hline Obj. Value & 84.0 & 85.2 & 80.5 \\
\hline
\end{tabular}

\subsection{Time and Cost Correlation}

As in the translator problem, the quality model for the software problem was expanded to allow correlation between cost and time for each task. The revised quality function is:

$$
Q(t, \mathrm{c})=K e^{-\left(\frac{t-\mu_{t}}{\sigma_{t}}\right)^{2}-2 \rho\left(\frac{t-\mu_{t}}{\sigma_{t}}\right)\left(\frac{c-\mu_{c}}{\sigma_{c}}\right)-\left(\frac{c-\mu_{c}}{\sigma_{c}}\right)^{2}}
$$

Table 10 Optimal Stochastic Solution for the Software Problem Assuming Triangular Probability Distributions with Varying Correlation Coefficients for Each Task

\begin{tabular}{|c|c|c|}
\hline \multirow{2}{*}{$\begin{array}{c}\text { Uncertain Task } \\
\text { Correlation Coefficient }\end{array}$} & \multicolumn{2}{|c|}{ Optimal Stochastic Solution } \\
\cline { 2 - 3 } & Expected Value & Standard Deviation \\
\hline 0 & 84.0 & 4.2 \\
\hline .1 & 84.0 & 4.3 \\
\hline .2 & 83.8 & 4.5 \\
\hline .3 & 83.6 & 4.6 \\
\hline .4 & 83.2 & 4.9 \\
\hline .5 & 82.7 & 5.2 \\
\hline .6 & 82.1 & 5.3 \\
\hline .7 & 81.3 & 5.5 \\
\hline .8 & 80.8 & 5.6 \\
\hline .9 & 79.3 & 5.9 \\
\hline
\end{tabular}


To investigate the effect of cost and time correlation on the optimal solution, the stochastic optimization model for the software problem using the natural log formulation was run for correlation values over the range $0,0.1,0.2, \ldots, 0.9$. The same correlation value was assumed for each task. The triangular probability distribution was used for the uncertain time and cost parameters. The results are shown in Table 10.

The results show that the optimal solution is sensitive to changes in correlation. As correlation increases, the optimal objective value decreases and the risk, as measured by standard deviation, increases.

\section{Conclusions and Further Research}

Quality is an important component of project management and can be incorporated within the project planning and scheduling process. A project planning model that explicitly includes a quality function can be applied to evaluate the tradeoffs between quality, time and cost. This model also determines the level of each task's scheduled duration and cost that best achieves the project's quality objective. In this paper we extend this previous work and formulate and solve the Stochastic Time Cost Quality Tradeoff Problem (STCQTP). The resulting stochastic optimization model enables us to determine how uncertainty about time and cost, modeled through the quality function parameters, affects the project quality achieved and the decisions about each task. Using two case studies with real data, a translation agency and a software development company, we show how uncertainty can have a substantial effect on the project quality and task decisions. We have shown that correlation between the time and cost, if present, can have a significant effect on the solution, by reducing the expected optimal quality and increasing the risk. The form of the probability distribution for the uncertain parameters in some cases can also have an effect on the optimal solution. In practical terms, it would typically not be realistic to subject all tasks to these types of analysis, but to focus on those few that are likely to be most affected by uncertainty in a way that would be most significant to the overall project. With our approach, we have demonstrated a procedure for optimal hedging against uncertainties of time and cost in project scheduling.

Future research could investigate whether changing the project quality objective to the arithmetic or geometric mean would lead to significantly different results. Another area of future research could include a probability of delay and, if it occurs, include stochastic time and cost parameters. For example, suppose there is a chance of a weather delay and, if it occurs, the time and cost quality function parameters are assumed to follow probability distributions. Future research could also investigate methods that can monitor and control quality during the project, similar to how we manage time and cost. Such methods will provide better balance in project management, emphasizing the nature and quality of the work completed, not just its schedule and budget.

\section{References}

1. Ahadian, B., Veisy, O., \& Azizi, V. (2016). "A multi-objective stochastic programming approach for project time, cost and quality trade-off problem (TCQTP)", Jordan Journal of Civil Engineering, Vol. 10(4), pp. 553 - 563. 
2. Afruzi, E. N., Najafi, A. A., Roghanian, E., \& Mazinani, M. (2014). “A multiobjective imperialist competitive algorithm for solving discrete time, cost and quality trade-off problems with mode-identity and resource-constrained situations", Computers and Operations Research, Vol. 50, pp. 80-96.

3. Amiri, M., \& Golozari, F. (2011). "Application of fuzzy multi-attribute decision making in determining the critical path by using time, cost, risk, and quality criteria", International Journal of Advanced Manufacturing Technology, Vol. 54(1-4), pp. $393-401$.

4. Babu, A. J. G., \& Suresh, N. (1996). "Project management with time, cost, and quality considerations", European Journal of Operational Research, Vol. 88(2), pp. $320-327$.

5. Brucker, P., Drexl, A., Mohring, R., Neumann, K., \& Pesch, E. (1999). "Resource-constrained project scheduling: Notation, classification, models, and methods", European Journal of Operational Research, Vol. 112(1), pp. 3-41.

6. Cristobal, J. R. S. (2013). "Critical path definition using multicriteria decision making: PROMETHEE method", Journal of Management in Engineering, Vol. 29(2), pp. $158-163$.

7. Demeulemeester, E. L., De Reyck, B., \& Herroelen, W. (2006). "The discrete time/resource trade-off problem in project networks: A branch-and-bound approach", IIE Transactions, Vol. 32(11), pp. $1059-1069$.

8. Floyd, M., Barker, K., Rocco, C., \& Whitman, M. (2017). "A multi-criteria decision analysis technique for stochastic task criticality in project management", Engineering Management Journal, Vol. 29(3), pp. 165 - 178.

9. Fu, F., \& Zhang, T. (2016). "A new model for solving time-cost-quality tradeoff problems in construction", PLOS One, https://journals.plos.org/plosone/article?id=10.1371/journal.pone.0167142

10. Ghodsi, R., Skandari, M. R., Allahverdiloo, M., \& Iranmanesh, S. H. (2009). “A new practical model to trade-off time, cost, and quality of a project", Australian Journal of Basic and Applied Sciences, Vol. 3(4), pp. 3741 - 3756.

11. Hosseini, S. A., Akbarpour, A., Ahmadi, H., \& Aminnejad, B. (2018). "Balance of cost, time, and quality related to construction projects regarding the reinforced concrete of underground structures using a meta-heuristic algorithm", Archives of Civil Engineering, Warsaw, Vol. 63(4), pp. 103 - 121.

12. IBM Corp. (2016). IBM SPSS Statistics for Windows, Version 24.0, Armonk, NY: IBM Corp.

13. Icmeli-Tukel, O., \& Rom, W. O. (1997). "Ensuring quality in resource constrained project Scheduling", European Journal of Operational Research, Vol. 103, pp. 493-496.

14. Kelly, J. J. E., \& Walker, M. R. (1959). Critical path planning and scheduling: An introduction. Ambler, PA: Mauchly Associates.

15. Kerzner, H. (2003). Project management: A systems approach to planning, scheduling, and controlling. th $^{\text {th }}$ ed. New York: Wiley.

16. Khang, D. B., \& Myint, Y. M. (1999). "Time, cost and quality trade-off in project management: A case study", International Journal of Project Management, Vol. 17(4), pp. 249 - 256. 
17. Kim, J. Y., Kang, C. W., Hwang, I. (2012). "A practical approach to project scheduling: considering the potential quality loss cost in the time-cost tradeoff problem", International Journal of Project Management, Vol. 30, pp. 264-272.

18. Liberatore, M. J., \& Pollack-Johnson, B., 2013. "Improving project management decision making by modeling quality, time, and cost continuously", IEEE Transactions on Engineering Management, Vol. 60(3), pp. 518 - 528.

19. LINDO Systems (2019). LINGO version 18.0, Chicago, IL: LINDO Systems.

20. Mahdiraji, H. A., Hajiagha, S. H. R., Hashemi, S. S., \& Zavadskas, E. K. (2016). "A grey multi-objective linear model to find critical path of a project by using time, cost, quality and risk parameters", Ekonomie a Management, Vol. 19(1), pp. $49-61$.

21. Malcolm, D. G., Rosenboom, J. H., Clark, C. E., \& Fazar, W. (1959). "Application of a technique for research and development project evaluation", Operations Research, Vol. 7(5), pp. $646-669$.

22. Mohammadipour, F., \& Sadjadi, S. J. (2016). "Project cost-quality-risk tradeoff analysis in a time-constrained problem", Computers \& Industrial Engineering, Vol. 95, pp. $111-121$.

23. Monghasemi, S. M. R., Nikoo, M. R., Fasaee, M. A. K., \& Adamowski, J. (2015). "A novel multi criteria decision making model for optimizing time-costquality trade-off problems in construction projects", Expert Systems with Applications, Vol. 42 (6), pp. 3089 - 3104.

24. Mungle, S., Benyoucef, L., Son, Y.-J., \& Tiwari, M. K. (2013). "A fuzzy clustering-based genetic algorithm approach for time-cost-quality trade-off problems: A case study of highway construction project", Engineering Applications of Artificial Intelligence, Vol. 26, pp. 1953-1966.

25. Orm, M., \& Jeunet, J. (2018). "Time cost trade-off problems: A survey exploring the assessment of quality", Computers \& Industrial Engineering, Vol. 118 , pp. $319-328$.

26. Pfeifer, J., Barker, K., Ramirez-Marquez, J. E., \& Morshedlou, N. (2015). "Quantifying the risk of project delays with a genetic algorithm", International Journal of Production Economics, Vol. 170, pp. 34 - 44.

27. Project Management Institute (2017). What is project management? https://www.pmi.org/about/learn-about-pmi/what-is-project-management. Accessed September 29, 2018.

28. Razavi, S. H., Akrami, H., Hashemi, S. S., \& Amoozad Mahdiraji, H. (2015). "An integer grey goal programming for project time, cost and quality tradeoff", Engineering Economics, Vol. 26(1), pp. 93 - 100.

29. Salmasnia, A., Mokhtari, H., \& Abadi, I. N. K. (2016). "A robust scheduling of projects with time, cost, and quality considerations", International Journal of Advanced Manufacturing Technology, Vol. 60, pp. 631 - 642.

30. Tareghian, H. R., \& Taheri, S. H. (2006). "On the discrete time, cost and quality trade-off Problem", Applied Mathematics and Computation, Vol. 181, pp. 13051312. 
31. Tareghian, H. R., \& Taheri, S. H. (2007). "A solution procedure for the discrete time, cost and quality tradeoff problem using electromagnetic scatter search", Applied Mathematics and Computation, Vol. 190, pp. 1136-1145.

32. Tiwari, V., Patterson, J. H., \& Mabert, V. A. (2009). "Scheduling projects with heterogeneous resources to meet time and quality objectives", European Journal of Operational Research, Vol. 193, pp. 780-790.

33. Tran, D.-H., Cheng, M.-Y., \& Cao, M.-T. (2015). "Hybrid multiple objective artificial bee colony with differential evolution for the time-cost-quality tradeoff problem", Knowledge-Based Systems, Vol. 74, pp. 176-186.

34. Tavana, M., Abtahi, A.-R., \& Khalili-Damghani, L. (2014). "A new multiobjective multi-mode model for solving the time-cost-quality trade-off project scheduling problem", Expert Systems with Applications, Vol. 41, pp. 1830 1846.

35. Zhang, L., Du, J., \& Zhang, S. (2014). "Solution to the time-cost-quality tradeoff problem in construction projects based on immune genetic particle swarm optimization", Journal of Management in Engineering, Vol. 30, pp. 163-172.

\section{About Our Authors}

Bruce Pollack-Johnson earned a B.A. in Sociology from Brandeis University, an M.A. in Applied Mathematics from Temple University, and an M.S. and Ph.D. in Operations Research from the University of Pennsylvania. He taught at Oberlin College, and is currently an Associate Professor of Mathematics and Statistics at Villanova University. He has published dozens of papers in project management, forecasting, educational modeling, and on teaching applied mathematics, as well as 3 editions of a two-volume text on business calculus and finite mathematics. His current research is on mathematical models incorporating quality considerations in project scheduling. He is a member of INFORMS.

Matthew J. Liberatore is the John F. Connelly Chair in Management at the Villanova School of Business (VSB), Villanova University. Dr. Liberatore received his Ph.D. in Operations Research from the Wharton School, University of Pennsylvania. He previously served VSB as the Associate Dean, Management Department Chair, and Founding Director of the Center for Business Analytics. Dr. Liberatore previously held management positions in operations analysis, information systems, and R\&D planning at FMC Corporation. Professor Liberatore has authored eleven books and published over 100 journal articles. His current research focuses on project management, supply chain planning, Business Intelligence, and virtual reality. 